ARRIGONI-BLANK, M. de F.; FAQUIN, V.; PINTO, J.E.B.P.; BLANK, A.F.; LAMEIRA, O.A. Adubação química e calagem em erva-baleeira. Horticultura Brasileira, Brasília, v. 17, n. 3, p. 211-215, novembro 1999.

\title{
Adubação química e calagem em erva-baleeira.
}

\author{
Maria de Fátima Arrigoni-Blank ${ }^{1}$; Valdemar Faquin ${ }^{2}$; José Eduardo B. P. Pinto ${ }^{2}$; Arie F. Blank ${ }^{1}$; Osmar \\ A. Lameira ${ }^{5}$. \\ ${ }^{1}$ UFS - DFS, Av. Marechal Rondon s/n, B. Rosa Elze, 49.100-000 São Cristóvão - SE; ${ }^{2}$ UFLA - DCS, C. Postal 37, 37.200-000 Lavras - \\ MG; ${ }^{5}$ Embrapa Amazonia Oriental, C. Postal 48, 66.095-100, Belém - PA.
}

\section{RESUMO}

Cordia verbenacea, popularmente conhecida como erva-baleeira, é uma planta medicinal perene com propriedade antiinflamatória. $\mathrm{O}$ trabalho teve como objetivo avaliar os efeitos da adubação química e da calagem no crescimento e na nutrição da erva-baleeira, mediante a técnica da diagnose por subtração. Foram utilizados no experimento mudas com 40 dias de idade após a emergência das sementes e, cultivadas em vasos com capacidade para $5 \mathrm{dm}^{3}$ em condições de casa de vegetação. Os tratamentos usados foram: completo (adubado com N, P, K, S, B, Cu, Fe, Zn e calagem); completo sem calagem; completo exceto cada nutriente (N, P, K, S, B, Cu, Fe, Zn) e testemunha (solo natural). Após 120 dias do transplantio observou-se que, no cultivo da erva-baleeira em solos ácidos e de baixa fertilidade, a calagem e a adubação são essenciais para o seu crescimento. No solo utilizado, os nutrientes que apresentaram maiores respostas quanto ao crescimento e nutrição da espécie foram o $\mathrm{N}, \mathrm{K}$ e B, bem como a calagem.

Palavras-chave: Cordia verbenacea, nutrição mineral, elemento faltante.

\begin{abstract}
Chemical fertilization and liming in Cordia verbenacea.

Cordia verbenacea is popularly known as 'erva-baleeira' and is a perennial medicinal plant with anti-inflammatory properties. The aim of this work was to evaluate the effects of chemical fertilization and liming on growth and nutrition of $C$. verbenacea, using the missing element technique. The experiment was conducted in greenhouse using 40 day old seedlings, which were cultivated into 5 $\mathrm{dm}^{3}$ pots. The treatments were: complete (fertilization with $\mathrm{N}, \mathrm{P}, \mathrm{K}$, $\mathrm{S}, \mathrm{B}, \mathrm{Cu}, \mathrm{Fe}, \mathrm{Zn}$ and liming); complete without liming; complete missing each nutrient (N, P, K, S, B, Cu, Fe, Zn) and control (natural soil). Evaluation was done 120 days after transplanting. Liming and fertilization are essential for $C$. verbenacea growth, when cultivated in poor acid soils. For the soil employed in this experiment the nutrients $\mathrm{N}, \mathrm{K}$ and $\mathrm{B}$, as well as liming, gave best results for $C$. verbenace $a$ growth and nutrition.
\end{abstract}

Keywords: Cordia verbenacea, mineral nutrition, missing element.

(Aceito para publicação em 04 de outubro de 1999)

\begin{abstract}
A erva-baleeira (Cordia verbenacea . - Boraginaceae) é uma planta medicinal perene, de 1 a $2 \mathrm{~m}$, podendo excepcionalmente atingir $3 \mathrm{~m}$ de altura, muito ramosa e flexível, com folhas tipicamente verrucosas, aromáticas, inflorescência espigosa com flores brancas e fruto vermelho quando maduro (Silva Jr. et al., 1995), que ocorre ao
\end{abstract}

longo de todo o litoral brasileiro. Os princípios ativos básicos da planta são os óleos essenciais, os flavonóides como a artemetina (Sertie et al., 1990) e isoflavonóides (Lameira, 1997) com atividades antiinflamatória.

A maioria dos solos brasileiros são ácidos, o que pode causar problemas em plantios comerciais. Além da alta satur ração de alumínio que pode ter efeitos tóxicos para as plantas, os solos ácidos normalmente contêm baixo teor de cálcio e de magnésio trocáveis, cátions de grande importância para o desenvolvimento radicular. Uma das alternativas para minimizar esse problema é a correção da acidez através da prática da calagem (Goedert et al., 1991). 
Na literatura há poucas informações sobre a fertilização química e exigências nutricionais de plantas medicinais, principalmente no Brasil. Raras vezes o emprego de adubos químicos, dentro dos limites técnicos, altera o teor dos princípios ativos das plantas. $\mathrm{O}$ aumento de biomassa pode compensar uma redução do teor de fitofármacos, mas depende da análise econômica, que deve ser feita em cada situação (Correa Jr. et al., 1991).

A adubação química em guaco (Mikanea glomerata), foi benéfica quando na aplicação de nitrogênio mineral (60 g de sulfato de amônio por planta) e resultou no aumento da produção de fitomassa em torno de seis vezes em relação à testemunha (Pereira et al., 1996). Na fertilização de plantas medicinais, avaliando o efeito de doses de NPK durante a fase de formação de mudas de jaborandi (Pilocarpus microphyllus Starf.), utilizando-se como substrato uma mistura de terra preta, serragem curtida e esterco de curral, na proporção de $3: 1: 1$, concluiu-se que as melhores produções de matéria seca foram conseguidas com as aplicações de 180 e $120 \mathrm{mg} / \mathrm{kg}$ de nitrogênio e fósforo, respectivamente (Brasil, 1996).

Para Atropa belladona a fertilização com 100, 35 e $120 \mathrm{~kg} / \mathrm{ha}$ de N, P e K, respectivamente, resultou no aumento de $750 \mathrm{~kg} / \mathrm{ha}$ para $1700 \mathrm{~kg} / \mathrm{ha}$ de folhas secas e não houve diferença significativa entre os teores de atropina, o que indica que a adubação química é vantajosa para esta espécie (Montanari Jr. et al., 1993).

Com a erva-baleeira o NPK em plantas adultas aumentou a produção de artemetina e hidroxiartemetina (Montanari Jr.et al., 1992).

Na busca de um sistema de produção economicamente viável, deve-se implantar um programa de nutrição na cultura. No caso da erva-baleeira ( $C$. verbenacea), tem-se pouca ou nenhuma informação disponível sobre suas exigências nutricionais e dos teores dos nutrientes na parte aérea. Assim, objetivou-se no presente trabalho, avaliar os efeitos da adubação química e da calagem no crescimento e na nutrição da erva-baleeira.

\section{MATERIAL E MÉTODOS}

O trabalho foi conduzido na casa de vegetação do Departamento de Agricul- tura da Universidade Federal de Lavras - UFLA, em vasos com $5 \mathrm{dm}^{3}$. Utilizou-se amostra da camada superficial $(0-20 \mathrm{~cm})$ de um Cambisolo álico, textura média, coletado no município de Itumirim - MG, com as seguintes características químicas: $\mathrm{pH}$ em água - 4,7; $\mathrm{P}$ - 2 e K - 39 mg/dm³ (Mehlich 1); Ca - 8, $\mathrm{Mg}-2$ e $\mathrm{Al}-7 \mathrm{mmol}_{\mathrm{c}} / \mathrm{dm}^{3}(\mathrm{KCl} 1$ mol.L-1) (EMBRAPA, 1979); S - $\mathrm{SO}_{4}^{2-}$ $11 \mathrm{mg} / \mathrm{dm}^{3}$ (Tedesco et al., 1985); Zn $11, \mathrm{Cu}-0,2, \mathrm{Fe}-65,4$ e $\mathrm{Mn} 9,3 \mathrm{mg} / \mathrm{dm}^{3}$ (DTPA) e B (água quente) - 0,13 mg/ $\mathrm{dm}^{3}$ (Jackson, 1970).

$\mathrm{O}$ delineamento experimental foi o de blocos ao acaso, com 10 tratamentos baseados na técnica do elemento faltante e 8 repetições. Os tratamentos foram: Completo (adubado com N, P, K, S, B, $\mathrm{Cu}, \mathrm{Fe}, \mathrm{Zn}$ e calagem); Completo sem calagem (-Cal); Completo sem N (-N); Completo sem P (-P); Completo sem K (-K); Completo sem S (-S); Completo sem B (-B); Completo sem Zn (-Zn); Completo sem $\mathrm{Fe}(-\mathrm{Fe})$ e Testemunha (solo natural). O tratamento completo não recebeu Mn porque encontrava-se em níveis elevados no solo. Para o cálculo da necessidade de calagem, empregou-se o método da saturação por bases, para elevar V a 70\%, usando-se $\mathrm{CaCO}_{3}$ e $\mathrm{MgCO}_{3}$ p.a., na proporção equivalente $\mathrm{Ca}: \mathrm{Mg}$ de 4:1 (Comissão..., 1989; Raij et al., 1996). Após a calagem, o solo foi mantido em incubação por 20 dias, com umidade de $70 \%$ do volume total de poros (VTP). Quando pertinente ao tratamento, foi aplicada a seguinte adubação básica de plantio: $67 \mathrm{mg} / \mathrm{dm}^{3}$ de $\mathrm{N} ; 150 \mathrm{mg} / \mathrm{dm}^{3}$ de $\mathrm{P} ; 97,5 \mathrm{mg} / \mathrm{dm}^{3}$ de $\mathrm{K} ; 40 \mathrm{mg} / \mathrm{dm}^{3}$ de $\mathrm{S} ; 0,5 \mathrm{mg} / \mathrm{dm}^{3}$ de B; $1,5 \mathrm{mg} / \mathrm{dm}^{3}$ de $\mathrm{Cu} ; 5,0 \mathrm{mg} / \mathrm{dm}^{3}$ de $\mathrm{Zn}$ e $5,0 \mathrm{mg} / \mathrm{dm}^{3}$ de Fe de solo. As fontes dos nutrientes foram reagentes p.a. $\left(\mathrm{NH}_{4} \mathrm{NO}_{3}, \mathrm{NH}_{4} \mathrm{H}_{2} \mathrm{PO}_{4}, \mathrm{~K}_{2} \mathrm{SO}_{4}, \mathrm{KH}_{2} \mathrm{PO}_{4}\right.$, $\mathrm{Ca}\left(\mathrm{H}_{2} \mathrm{PO}_{4}\right)_{2} \cdot \mathrm{H}_{2} \mathrm{O}, \quad \mathrm{CaSO}_{4} \cdot 2 \mathrm{H}_{2} \mathrm{O}$, $\mathrm{MgSO}_{4} \cdot 7 \mathrm{H}_{2} \mathrm{O}, \mathrm{H}_{3} \mathrm{BO}_{3}, \mathrm{CuCl}_{2}, \mathrm{ZnCl}_{2}$, Fe-EDTA).

Duas adubações de cobertura foram realizadas. A primeira, aos 40 dias após plantio (DAP), aplicaram-se $15 \mathrm{mg} / \mathrm{dm}^{3}$ de $\mathrm{N}$, exceto nos tratamentos $-\mathrm{N}$ e solo natural, e $20 \mathrm{mg} / \mathrm{dm}^{3}$ de $\mathrm{P}$ no tratamento -P. Essa dose de $\mathrm{P}$ foi necessária para obtenção de material vegetal para as análises químicas e dos princípios ativos. A segunda, aos 70 DAP, constou da aplicação de: $20 \mathrm{mg}$ de N, 32,8 mg de $\mathrm{Mg}, 114 \mathrm{mg}$ de $\mathrm{Ca}, 32 \mathrm{mg}$ de $\mathrm{S} / \mathrm{dm}^{3}$ de solo, exceto na testemunha e nos tratamentos onde foram omitidos.

As mudas de erva-baleeira (Cordia verbenacea $\mathrm{L}$.) foram obtidas de sementes germinadas em bandejas com areia. Aos 40 dias da emergência transplantou-se uma única muda por vaso, mantendo-se a umidade na capacidade de campo. As plantas foram colhidas aos 120 dias após o transplantio, separando-se em folhas, ramos e raízes, secas em estufa a $70^{\circ} \mathrm{C}$, exceto as folhas, que foram secas a $45^{\circ} \mathrm{C}$, em virtude da posterior análise do princípio ativo, tomando-se em seguida, o peso da matéria seca de cada parte da planta. Os teores dos macro e micronutrientes na matéria seca da parte aérea (caule e folha) foram analisados quimicamente: o $\mathrm{N}$, pelo método de Kjeldahl; o $\mathrm{P}, \mathrm{K}, \mathrm{Ca}$, $\mathrm{S}, \mathrm{Fe}$ e $\mathrm{Zn}$, pela digestão nitricoperclórica, e a determinação no extrato foi feita como segue: $\mathrm{P}$ colorimetria; $\mathrm{K}$ - fotometria de chama; $\mathrm{S}$ - turbidimetria; $\mathrm{Ca}$, Fe e $\mathrm{Zn}$ espectrofotometria de absorção atômica e o B por incineração e determinação colorimétrica pelo método da curcumina (Malavolta et al., 1989).

Foram realizadas análises de variância da matéria seca de cada parte e do total da parte aérea, das raízes, altura de planta, teores dos macro e micronutrientes, sendo as médias comparadas pelo teste de Tukey, de acordo com Gomes (1985).

\section{RESULTADOS E DISCUSSÃO}

\section{Altura e peso de matéria seca}

A omissão da calagem (-Cal), do B (-B) e $\mathrm{K}(-\mathrm{K})$ reduziram significativamente a produção de matéria seca de raiz (exceto no -K), folhas, caule e parte aérea total da espécie. $\mathrm{O}$ tratamento $-\mathrm{N}$ apresentou uma redução somente no peso da matéria seca de folhas e parte aérea total (Tabela 1). Por outro lado, os tratamentos com omissão do $\mathrm{S}(-\mathrm{S})$, $\mathrm{Zn}(-\mathrm{Zn}), \mathrm{Fe}$ (-Fe) e do $\mathrm{P}(-\mathrm{P})$ não promoveram nenhum efeito sobre o crescimento da espécie. Vale ressaltar que o $\mathrm{P}$ não foi totalmente omitido da adubação, em virtude da sua aplicação em cobertura aos 40 dias após o transplante. Esse 
Tabela 1. Altura de planta, peso da matéria seca de raiz, caule, folha e produção relativa da parte aérea de Cordia verbenacea, em função dos tratamentos. Lavras, UFLA, 1996.

\begin{tabular}{|c|c|c|c|c|c|c|}
\hline \multirow{2}{*}{ Tratamento } & \multirow{2}{*}{$\begin{array}{l}\text { Altura de } \\
\text { Planta }\end{array}$} & \multicolumn{4}{|c|}{ Peso da matéria seca } & \multirow{2}{*}{$\begin{array}{l}\text { Produçãc } \\
\text { Relativa1 }\end{array}$} \\
\hline & & Raiz & Caule & Folha & Parte aérea & \\
\hline & $\mathbf{c m}$ & & $g / p$ & & & (\%) \\
\hline Completo & $35,83 a b c$ & $7,00 \mathrm{ab}$ & $4,41 a$ & $7,39 a b$ & $11,81 \mathrm{a}$ & 100 \\
\hline- Cal & $19,50 \quad \mathrm{e}$ & $3,74 \quad d$ & $2,02 \mathrm{~cd}$ & $5,42 \quad c$ & $7,45 \mathrm{~cd}$ & 63 \\
\hline$-\mathrm{N}$ & $28,30 \mathrm{~cd}$ & $7,82 \mathrm{a}$ & $3,69 a b$ & $5,25 \quad c$ & $8,94 \mathrm{bcd}$ & 76 \\
\hline$-P$ & $36,50 a b$ & $6,42 a b$ & $3,92 a b$ & $6,33 a b c$ & $10,26 a b$ & 87 \\
\hline$-K$ & $34,18 a b c$ & 5,85 bc & $2,96 \mathrm{bc}$ & 5,51 & $8,47 \mathrm{bcd}$ & 72 \\
\hline$-S$ & $30,20 \quad b c$ & $6,10 a b c$ & $4,10 a b$ & $7,65 a$ & $11,76 \mathrm{a}$ & 99 \\
\hline$-B$ & $28,29 \mathrm{~cd}$ & 3,41 & $1,92 \mathrm{~cd}$ & $5,47 \quad c$ & $7,40 \quad d$ & 63 \\
\hline$-\mathrm{Zn}$ & $35,64 a b c$ & $7,81 \mathrm{a}$ & $4,10 a b$ & $6,05 \mathrm{bc}$ & $10,16 a b c$ & 86 \\
\hline$-\mathrm{Fe}$ & 38,70 a & $7,50 a b$ & $4,21 a b$ & $6,30 a b c$ & $10,51 a b$ & 89 \\
\hline Testemunha & 21,75 & $4,42 \mathrm{~cd}$ & 1,03 & 2,55 & 3,59 & 30 \\
\hline
\end{tabular}

Médias seguidas pela mesma letra nas colunas não diferem entre si (Tukey $5 \%$ ).

1/ $\mathrm{PR}(\%)=[\mathrm{MS}($ Trat. $) / \mathrm{MS}$ (completo) $] \times 100$

fato deve-se à total paralisação do crescimento e dos sintomas visuais típicos de deficiência de fósforo observado nas plantas do tratamento -P, nesse período. Assim, visando a obtenção de material vegetal para as análises químicas e dos princípios ativos, decidiu-se pela aplicação de $20 \mathrm{mg} / \mathrm{dm}^{3}$ de $\mathrm{P}$ no tratamento de sua omissão. Mas, a dose de P aplicada, promoveu uma grande resposta das plantas em crescimento e produção de matéria seca (Tabela 1).

De acordo com a Comissão de Fertilidade do Solo do Estado de Minas Gerais (1989) e Raij et al. (1996), o solo utilizado no experimento é ácido, com baixos níveis de $\mathrm{K}, \mathrm{P}, \mathrm{Ca}, \mathrm{Mg}$ e $\mathrm{S}$ e com níveis de $\mathrm{Al}$ e $\mathrm{Mn}$ elevados. Mesmo assim, os resultados da Tabela 1, mostram que a omissão da calagem e dos nutrientes da adubação, não promoveu efeitos drásticos no crescimento e produção de matéria seca da espécie. Essa observação, associada à grande resposta da planta à pequena dose de $\mathrm{P}$ aplicada em cobertura $\left(20 \mathrm{mg} / \mathrm{dm}^{3}\right)$, permite inferir que a erva-baleeira é uma espécie de pequena exigência nutricional e/ou apresenta elevada eficiência de absorção e utilização dos nutrientes. Também, que a adubação básica utilizada no presente experimento e aquelas sugeridas para vasos (Malavolta, 1980 e Raij et al., 1996), certamente, são doses excessivas para essa espécie. Interessante ressaltar, também, que de acordo com Raij et al. (1996), os teores dos micronutrientes encontram-se na análise química do solo: B - baixo, Fe - alto, Mn - alto e Zn - médio. Portanto, a resposta da planta às suas omissões, está de acordo com a classificação citada.

Teores dos nutrientes na matéria seca

As espécies medicinais fazem parte de um grupamento de plantas pouco estudado e praticamente inexistem informações sobre adubação e nutrição dessas espécies e no caso de suspeitas de distúrbios nutricionais recomenda-se utilizar técnicas de análises de micronutrientes em solos, bem como a diagnose foliar comparativa, usando amostras pareadas (com e sem problemas), para análise química de macro e micronutrientes (Raij et al., 1996). Assim, a Tabela 2 mostra os teores dos nutrientes no caule e nas folhas da erva-baleeira, nos tratamentos nos quais foram omitidos (-M), comparativamente aos valores obtidos no tratamento completo (Compl.).

Observa-se que, de maneira geral, houve uma tendência de os teores, dos nutrientes serem maiores no tratamento completo (Compl.) em relação àqueles onde foram omitidos (-M), porém, nem sempre significativamente. As omissões de $\mathrm{B}, \mathrm{K}$ e $\mathrm{N}$, bem como a omissão da calagem, foram aquelas que mais influenciaram a produção relativa (PR) do caule e das folhas e, à exceção do $\mathrm{B}$ no caule, os teores no tratamento -M, foram sempre inferiores ao completo, indicando que os mesmos encontram-se em níveis abaixo do adequado. Ressalta-se que foram observados sintomas visuais típicos de deficiência desses nutrientes nos tratamentos nos quais foram omitidos (-M). Os tratamentos $-\mathrm{S},-\mathrm{Zn},-\mathrm{Fe}$ e -B, no caule, não diferiram significativamente do tratamento completo, enquanto que na folha, somente o - Zn foi significativamente igual ao completo.

É interessante observar que a omissão de B (-M) promoveu uma drástica redução da PR do caule (44\%), com teores do micronutriente significativamente igual ao tratamento completo (Tabela 2). Segundo Malavolta et al. (1989), esse fato é explicado pelo efeito "Steenyberg", que ocorre em casos onde o nutriente está muito deficiente no solo e a sua aplicação na adubação promove uma resposta muito grande da planta em crescimento, o que pode ser visto na Tabela 1, comparando-se a matéria seca do caule do tratamento -B com o completo. Nesse caso, o rápido incremento na produção de massa vegetal, proporcionalmente maior à absorção do nutriente, não permite o aumento na sua 
Tabela 2. Teores de macro e micronutrientes no caule e folhas no tratamento completo (Compl.) e nos respectivos tratamentos de omissão (-M), e produção relativa (PR) de matéria seca de caule e folhas do tratamento de omissão em comparação ao completo, de Cordia verbenacea. Lavras, UFLA, 1996.

\begin{tabular}{|c|c|c|c|c|c|c|c|c|c|}
\hline \multirow{2}{*}{ Nutriente } & \multicolumn{4}{|c|}{ Caule } & \multicolumn{5}{|c|}{ Folha } \\
\hline & Compl. & & $-M$ & PR & Compl. & & -1 & & PR1/ \\
\hline & \multicolumn{3}{|c|}{ g.kg-1 } & $\%$ & \multicolumn{4}{|c|}{ g kg-1 } & $\%$ \\
\hline $\bar{N}$ & 15,35 & $a$ & $8,65 \quad b$ & 84 & 30,35 & $a$ & 20,05 & $b$ & 71 \\
\hline$P$ & 2,25 & $a$ & $2,00 a$ & 89 & 2,12 & a & 2,10 & $a$ & 86 \\
\hline K & 10,57 & $a$ & $3,42 \quad b$ & 67 & 20,90 & a & 4,10 & $b$ & 74 \\
\hline Ca (calagem) & 0,072 a & & $0,049 \quad b$ & 46 & 0,0035 & a & 0,00 & $9 b$ & 73 \\
\hline Mg (calagem) & 0,076 & & $0,044 \quad b$ & 46 & 0,00054 & $a$ & 0,000 & $25 \mathrm{~b}$ & 73 \\
\hline \multirow[t]{2}{*}{$S$} & 1,32 & a & $0,92 a$ & 93 & 2,22 & $a$ & 1,45 & $b$ & 103 \\
\hline & \multicolumn{3}{|c|}{ mg.kg-1 } & $\%$ & \multicolumn{4}{|c|}{$\mathrm{mg} \cdot \mathrm{kg}-1$} & $\%$ \\
\hline $\bar{B}$ & 18,66 & $\bar{a}$ & $20,03 a$ & 44 & 58,27 & $\bar{a}$ & 43,08 & $\mathrm{~b}$ & 74 \\
\hline $\mathrm{Zn}$ & 17,49 & a & 11,41 a & 93 & 37,94 & a & 22,38 & $a$ & 82 \\
\hline $\mathrm{Fe}$ & $96,51 \quad c$ & a & 66,21 a & 95 & 374,46 & a & 302,05 & $b$ & 85 \\
\hline
\end{tabular}

Médias seguidas pela mesma letra nas linhas, para cada órgão, não diferem entre si (Tukey 5\%).

1/ $\mathrm{PR}(\%)=[\mathrm{MS}(-\mathrm{M}) / \mathrm{MS}(\mathrm{Compl})] \times$.100 .

concentração no tecido, podendo inclusive, ocorrer a diluição do mesmo. Já para as folhas este fato não foi observado. Possivelmente, devido a uma menor diferença na produção de matéria seca foliar entre o tratamento completo e o de omissão de B (Tabela 1). Mas, também, pela maior atividade metabólica e fisiológica das folhas do tratamento completo. Sabe-se que o B é um elemento imóvel na planta e seu transporte é feito de forma unidirecional no xilema, via corrente transpiratória (Marschner, 1995). Dessa maneira, o B encontrou melhores condições de ser transportado das raízes para a parte aérea no tratamento completo, apresentando, comparativamente ao tratamento de sua omissão (-M), maiores teores nas folhas e menores no caule (Tabela 2).

Devido à carência de informações sobre a nutrição mineral da erva-baleeira, os teores foliares dos nutrientes no tratamento completo (Tabela 2), podem ser usados, pelo menos como um referencial, na avaliação do estado nutricional da espécie, pela diagnose foliar. Embora, como já discutido, acredita-se que as doses de alguns nutrientes na adubação básica, tenham sido excessivas. É importante lembrar, também, que os teores foliares variam com as condições e épocas de cultivo, órgão analisado, idade do órgão e da planta, dentre outros.
Conclui-se que, após 120 dias do transplantio, no cultivo da erva-baleeira em solos ácidos e de baixa fertilidade, a calagem e a adubação são essenciais para seu crescimento. No solo utilizado, a falta da calagem e dos nutrientes $\mathrm{N}$, K e B causaram as maiores quedas na produção de folhas, órgão usado na medicina popular e que contém os princípios ativos. Quanto ao fósforo, os autores sugerem a realização de um estudo de curvas de resposta para obter dados precisos sobre a exigência nutricional dessa espécie quanto ao $\mathrm{P}$.

\section{AGRADECIMENTOS}

Os autores agradecem ao $\mathrm{CNPq}$ RHAE e a UFLA - DAG pela oportunidade de execução deste trabalho.

\section{LITERATURA CITADA}

BRASIL, E.C. Níveis de nitrogênio, fósforo e potássio na produção de mudas de jaborandi. REUNIÃO BRASILEIRA DE FERTILIDADE DO SOLO E NUTRIÇÃO DE PLANTAS, 22., 1996, Manaus. Anais ... Manaus: SBCS, 1996. p. 666-667.

COMISSÃO DE FERTILIDADE DO SOLO DO ESTADO DE MINAS GERAIS. Recomendações para o uso de corretivos e fertilizantes em Minas Gerais. 4 Aproximação. Lavras: CFSEMG, 1989. $176 \mathrm{p}$.

CORREA Jr., C.; MING, L.C.; SCHEFFER, M.C Cultivo de plantas medicinais, condimentares $e$ aromáticas. Curitiba: EMATER-PR, 1991. $151 \mathrm{p}$.
EMBRAPA - Serviço Nacional de Levantamento e Conservação dos Solos. Manual de métodos de análise de solo. Rio de Janeiro: EMBRAPA, 1979. n. p.

GOEDERT, W.J.; SOUZA, D.M.G. de; SCOLARI, D.O.G. Critérios para recomendação de calagem e adubação. In: OLIVEIRA, A.J.; GARRIDO, W. E.; ARAUJO, J.D.; LOURENÇO, S. (Coord.). Métodos de pesquisa em fertilidade do solo. Brasília: EMBRAPA SEA, 1991. p. 363-392.

GOMES, F.P. Curso de estatística experimental. São Paulo: Nobel, 1985. 466 p.

LAMEIRA, O.A. Propagação in vitro $e$ in vivo, dinâmica de crescimento de células, nutrição $e$ identificação de flavonóides em erva-baleeira (Cordia verbenacea $L$.). Lavras: UFLA, 1997. 88 p. (Tese doutorado).

JACKSON, M.L. Analisis quimico de suelos. Barcelona: Omega, 1970. 662 p.

MALAVOTA, E. Elementos de nutrição mineral de plantas. Piracicaba: ESALQ, 1980. $251 \mathrm{p}$

MALAVOLTA, E.; VITTI, G.C.; OLIVEIRA, S.A. de. Avaliação do estado nutricional das plantas: princípios e aplicações. Piracicaba: POTAFOS, 1989. 201 p.

MARSCHNER,H. Mineral nutrition of higher plants. New York: Academic Press, 1995. 889 p.

MONTANARI Jr., I.; FIGUEIRA, G.M.; MAGALHÃES, P.M. de; RODRIGUES, N. Influência da fertilização NPK na biomassa e no teor de alcalóide de Atropa belladona Linn. Revista Brasileira de Fisiologia Vegetal, Brasília, v. 5, n. 1, p. 71, 1993.

MONTANARI Jr., I.; MAGALHÃES, P.M. de; FIGUEIRA, G.M.; FOGLIO, M.A.; RODRIGUES, R.A.F.; HOPPEN, V.R.; SHARAPIN, N. Aspectos agronômicos e fitoquímicos do cultivo da baleeira. In: SIMPÓSIO DE PLANTAS MEDICINAIS DO BRASIL, 12., 1992, Curitiba. Anais ... Curitiba: UFPR, 1992. p. 180. 
PEREIRA, A.M.S.; MENEZES JÚNIOR, A.; CÂMARA, F.L.A.; FRANÇA, S.C. Efeito da adubação na produção de biomassa de Mikanea glomerata (guaco). In: SIMPÓSIO DE PLANTAS MEDICINAIS DO BRASIL, 14., Florianópolis, 1996. Anais..., Florianópolis: UFSC, 1996.
RAIJ, J.B. van; CANTARELLA, H.; QUAGGIO, J.A.; FURLANI, A.M.C. (eds.) Recomendações de adubação e calagem para o Estado de São Paulo. Campinas: IAC, 1996. 258 p. (Boletim Técnico, 100).

SERTIE, J.A.A.; BASILE, A.C.; PANIZZA, S. MATIDA, A.K.; ZELNIK, R. Antiinflamatory activity and sub-ocute toxicity of artemetin. Planta Medica, Stuttgart, v. 56, n. 11, p. 36 - 40, 1990.
SILVA JÚNIOR, A.A., VIZZOTO, V.J., GIORGI, E., MACEDO, S.G.; MARQUES, L.F. Plantas medicinais; caracterização e cultivo. Florianópolis: EPAGRI, 1995. 71 p. (Boletim Técnico, 68).

TEDESCO, M.J.; VOLKWEISS, S.T. BOHNEM, H. Análise de solo, plantas e outros materiais. Porto Alegre: UFRGS, 1985. (Boletim Técnico, 5). 